\title{
Right atrial spontaneous contrast: echocardiographic and clinical features
}

\author{
Michael A. DeGeorgia, ${ }^{1}$ Marc I. Chimowitz, ${ }^{2, *}$ Anne Hepner ${ }^{3}$ \& William F. Armstrong ${ }^{3}$ \\ Departments of Internal Medicine ${ }^{1}$, Neurology ${ }^{2} \&$ Cardiology ${ }^{3}$, University of Michigan Medical Center, Ann \\ Arbor, MI 48109, USA (*requests for offprints)
}

September 6, 1994

Key words: transesophageal echocardiography, right atrial spontaneous contrast, paradoxical embolism, stroke

\begin{abstract}
We describe the clinical and echocardiographic findings in eight patients with right atrial spontaneous echo contrast who were identified from 648 consecutive patients undergoing transesophageal echocardiography. Common findings in these patients were right atrial enlargement ( 8 patients), tricuspid regurgitation ( 7 patients), atrial fibrillation or flutter (6 patients), elevated right ventricular pressure (5 patients), moderate or severe mitral valve disease ( 5 patients), and right to left interatrial shunts ( 3 patients). Right heart catheterization in three patients showed markedly elevated right atrial, right ventricular, and pulmonary artery pressures. Two patients had thromboembolic events - one patient had recurrent pulmonary emboli, and another patient with an atrial septal aneurysm had recurrent transient ischemic attacks. Right atrial echo contrast is an uncommon finding at echocardiography that is associated with severe right heart dysfunction. It may also be associated with paradoxical or pulmonary embolism.
\end{abstract}

\section{Introduction}

Spontaneous echo contrast (SEC) is a dynamic smokelike signal that is detected by transesophageal echocardiography (TEE) in patients with stasis of blood in one or more of the cardiac chambers. SEC is most commonly visualized in the left atrium in patients with atrial fibrillation or mitral stenosis, but it is also seen occasionally in the right atrium or left ventricle.

The echocardiographic and clinical features associated with left atrial SEC are well described [1-3], however, there are limited data on the features associated with right atrial SEC. In this article, we describe the clinical and echocardiographic features of eight patients with right atrial SEC.

\section{Methods}

Patients with right atrial SEC were identified from consecutive patients undergoing TEE at the University of Michigan Medical Center between January 1990 and December 1991. Most of the patients with right atrial SEC on TEE also underwent transthoracic echocardio- graphy (TTE). TEE was performed using either an ATL Ultramark 9 system (Bothwell, WA) equipped with a $5 \mathrm{MHz}$ biplane transesophageal probe, or an Acuson 128XP system (Mountainview, CA) equipped with a $5 \mathrm{MHz}$ monoplane transesophageal probe. Spontaneous contrast was diagnosed if smoke-like echoes that swirled in a circular pattern within any of the cardiac chambers were identified. Differentiation of spontaneous contrast from excessive gain echoes was achieved by decreasing the gain settings.

Transesophageal and transthoracic echocardiographic features of patients with right atrial SEC were evaluated by two experienced echocardiographers (WFA and AH). Using TTE, the anteroposterior left atrial diameter was measured at end-systole from the parasternal long-axis view [4]. Another measurement of left atrial size was obtained using TEE by measuring the largest diameter of the left atrium in the 4-chamber view. Right atrial size was obtained by measuring the largest diameter of the right atrium in the 4-chamber view using TEE. The size and contractility of the right atrial appendage were also assessed - since there is no standard method for measuring right atrial appendage size, we subjectively graded the right atrial 
appendage as normal or large based on the impression of the echocardiographers.

Using TEE, ventricular function (right and left) was subjectively classified as normal or mildly, moderately, or severely depressed. Mitral and tricuspid regurgitation were graded using a semiquantitative scale $(0-+3)$ based on color flow imaging [5,6]. An interatrial septal aneurysm was diagnosed if the atrial septum appeared abnormally redundant and protruded into either atrium by $>1.1 \mathrm{~cm}^{7}$. Classification of the type of atrial septal aneurysm was based on whether the aneurysm protruded into the left or right atrium or both: type $1 \mathrm{~A}$ - continuous protrusion into the right atrium, type $1 \mathrm{~B}$ predominant protrusion into the right atrium with phasic excursion into the left atrium, type 11 - persistent protrusion into the left atrium $[7,8]$. All patients with right atrial SEC were evaluated for a right to left shunt across the interatrial septum after intravenous injection of agitated saline [7]. A right to left shunt was diagnosed if microbubbles were visualized in the left atrium immediately after their appearance in the right atrium.

The medical records of all patients with right atrial SEC were reviewed to determine the presence of the following clinical features: age, hypertension, smoking, diabetes, atrial fibrillation, valvular heart disease, myocardial infarction, cardiomyopathy, congestive heart failure, pulmonary embolus, transient ischemic attack (TIA), stroke, or peripheral embolus. Prospective data on clinical outcome were obtained from follow-up outpatient visits or telephone contact.

\section{Results}

Number of patients and clinical features

Of 648 patients undergoing TEE during the two year study period, eight patients $(1.2 \%)$ had right atrial SEC and 50 patients $(7.7 \%)$ had left atrial SEC. The clinical features of the eight patients with right atrial SEC are shown in the table.

Indications for TEE were evaluation of mitral valve disease ( 5 patients), congestive heart failure (1 patient), TIA (1 patient), and pulmonary embolus ( 1 patient). Five patients were in atrial fibrillation and one patient was in atrial flutter; five patients had a history of congestive heart failure; four patients had a history of myocardial infarction; one patient (patient 3) had an idiopathic cardiomyopathy.
Echocardiographic and cardiac catheterization findings

The echocardiographic findings are shown in the table. Four patients had right atrial SEC alone (patients 1-4), three patients had right and left atrial SEC (patients 5 7), and one patient with a mitral valve prosthesis had SEC in both atria and the left ventricle (patient 8 ).

Three patients had moderate or severe mitral stenosis, two patients had moderate or severe mitral regurgitation, four patients (patients 1,3,7,8) had moderate or severe left ventricular dysfunction, and seven patients had moderate or severe tricuspid regurgigation. Left atrial and right atrial diameters were increased (diameter $>40 \mathrm{~mm}$ ) in each of the eight patients with right atrial SEC. The size of the right atrial appendage tended to reflect the size of the right atrium i.e. the right atrial appendage was graded as large in three of the four patients with right atrial diameters exceeding $58 \mathrm{~mm}$ (patients 2,3,5), whereas the size of the right atrial appendage was considered normal in four patients with right atrial diameters less than $58 \mathrm{~mm}$. Rythmic contractions of the right atrial appendage were seen in only one of eight patients with right atrial SEC (patient 2). Six of seven patients without contractions of the right atrial appendage were in atrial fibrillation or atrial flutter.

A right to left interatrial shunt was present in three patients, and one patient had an atrial septal aneurysm without an apparent shunt by color flow or bubble contrast. One patient (patient 6) had Lutembacher's syndrome (mitral stenosis and an atrial septal defect). Thrombus was visualized in the left atrium in two patients, left ventricle in one patient, and right atrium in one patient (Fig. 1).

Right heart catheterization was performed in three patients, all of whom had markedly elevated right atrial, right ventricular, and pulmonary artery pressures (table).

\section{Thromboembolic events}

Patient 2, a 48 year old male with a history of a pulmonary embolus and an antero-septal myocardial infarction, presented in coma. An arterial blood gas revealed hypoxemia with a high pulmonary alveolar to arterial oxygen gradient. A toxic screen identified cocaine in his urine. A lung ventilation/perfusion scan showed three large areas of mismatch. Pulmonary angiography showed multisegmental filling defects. Venous ultrasonography and venography of the low- 

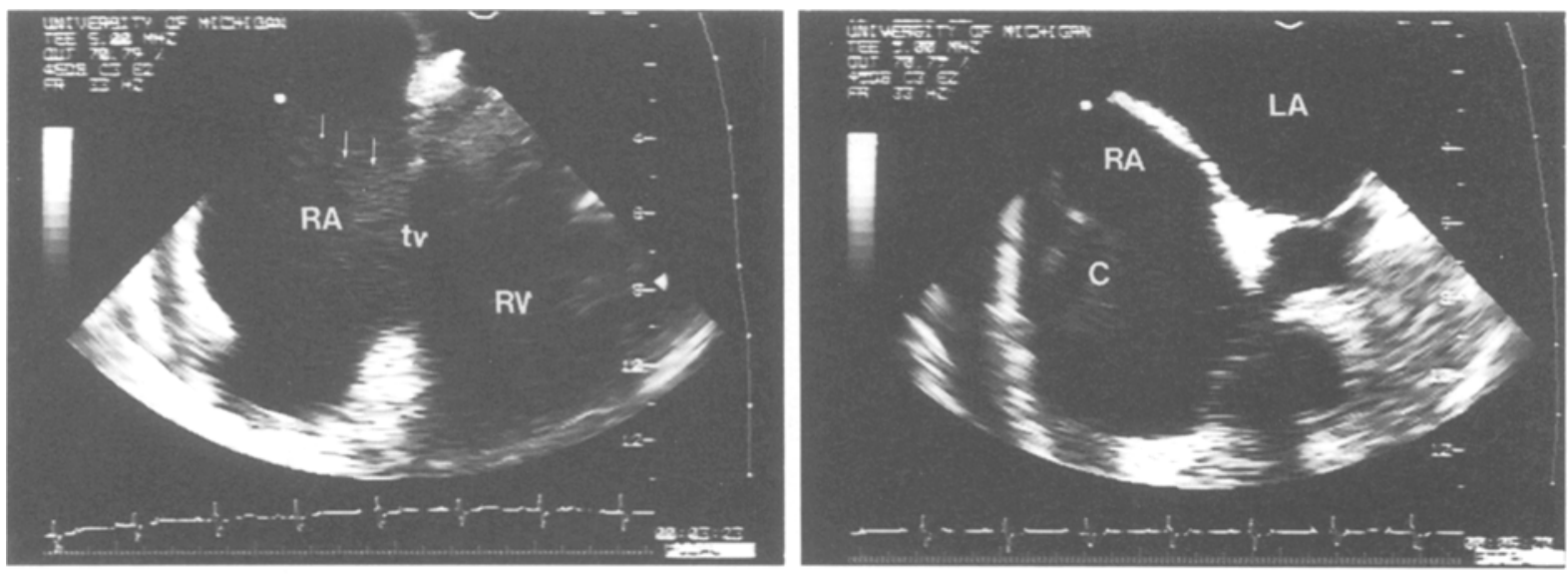

a

Fig. 1. Transesophageal echocardiography findings in patient 3 who had an idiopathic cardiomyopathy. (a) transverse plane view shows large right atrium (RA) with spontaneous contrast (arrows). $(b)$ another transverse plane view shows large right atrium (RA) with clot (C). LA $=$ left atrium, $t v=$ tricuspid valve, $R V=$ right ventricle.
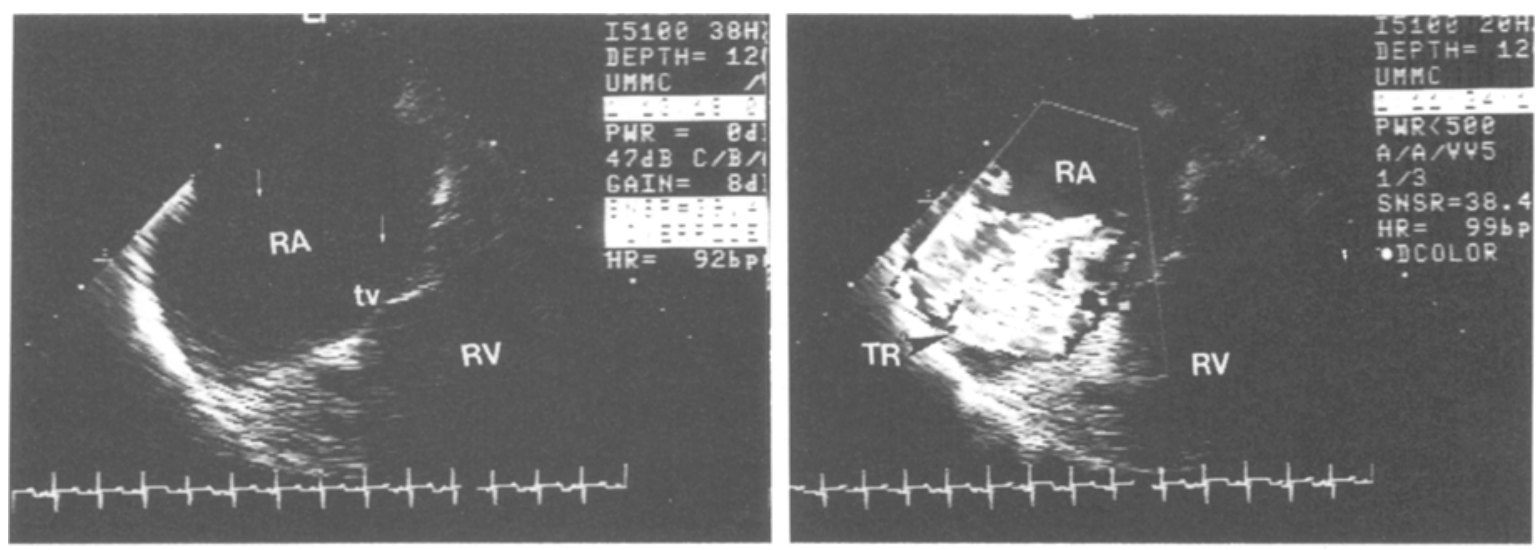

a

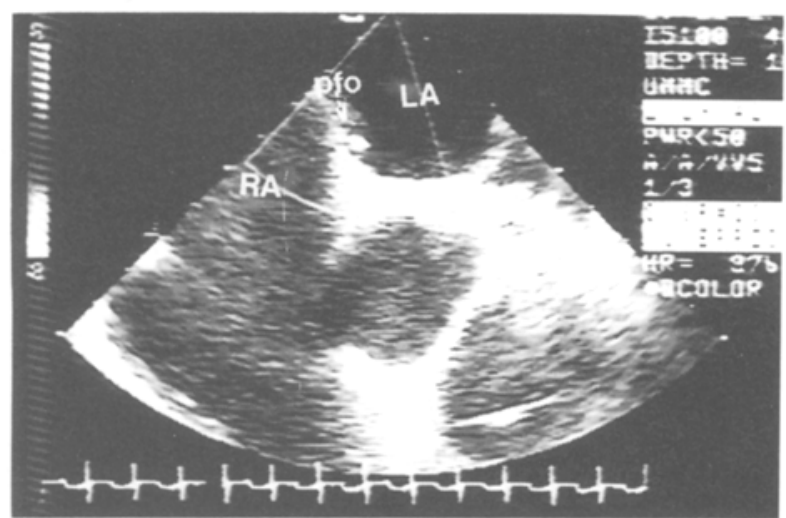

Fig. 2. Transesophageal echocardiography findings in patient 2 who presented with multiple pulmonary emboli. (a) transverse plane view shows massive enlargement of right atrium (RA) with spontaneous contrast (arrows). Color flow imaging (b) shows severe tricuspid regurgitation (TR with arrow). A view of the interatrial septum (c) shows a right to left shunt through a patent foramen ovale (pfo) by color flow imaging. t $v=$ tricuspid valve, $R V=$ ventricle, $L A=$ left atrium. 


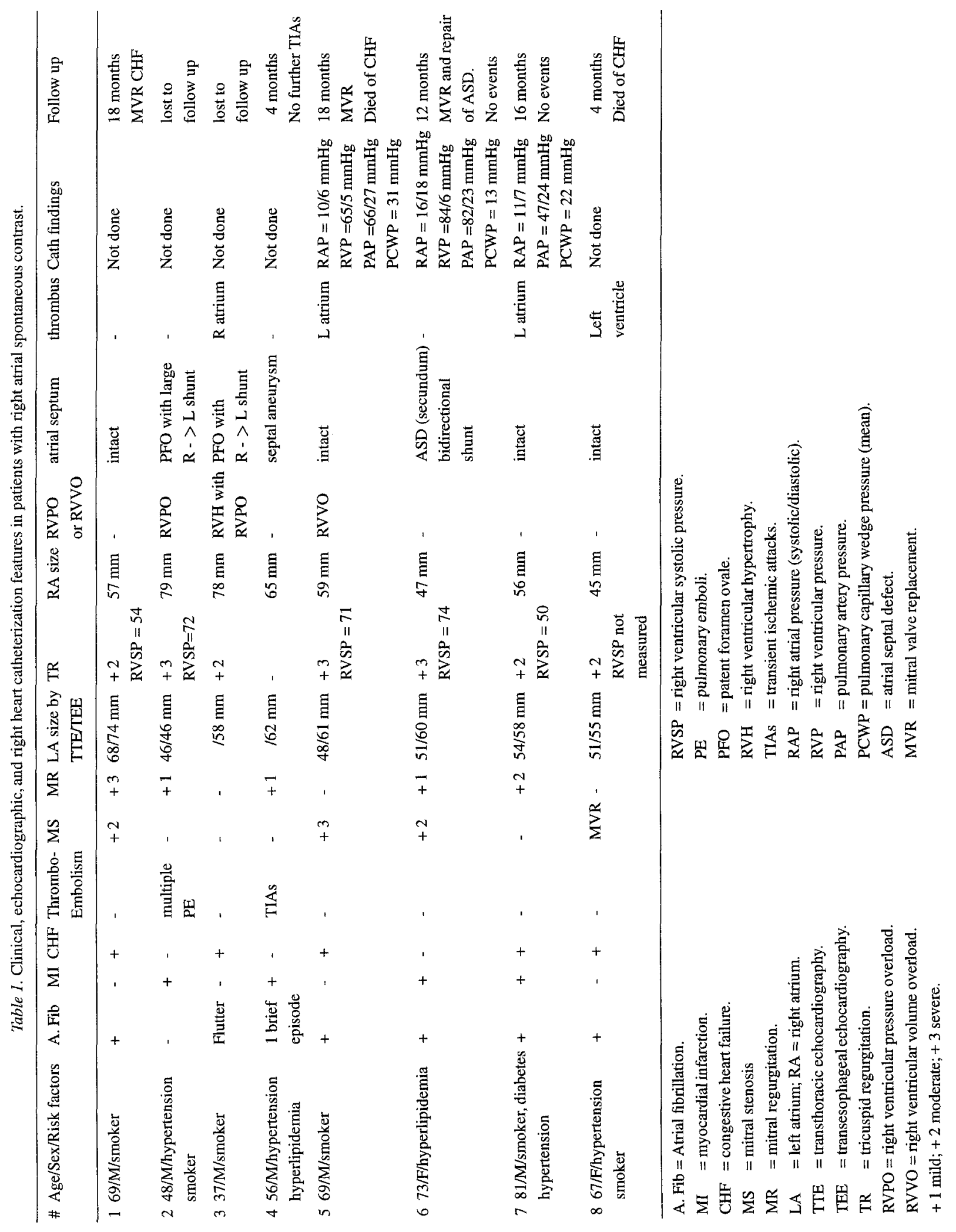


er extremities were normal, nevertheless, a Greenfield filter was inserted into the inferior vena cava.

TEE showed massive right atrial enlargement, severe tricuspid regurgitation, right atrial SEC, and a right to left shunt through a patent foramen ovale (Fig. 2). Left ventricular function and the structure and function of the pulmonary, mitral and aortic valves were normal. The patient was discharged a month after admission, did not return for follow up, and could not be contacted.

Patient 4, a 56 year old man with a history of hypertension and myocardial infarction, presented after four episodes of speech arrest and right arm weakness. Each of the episodes lasted less than five minutes. Computerized tomography and magnetic resonance imaging of the brain were normal. Carotid angiography showed no evidence of extracranial or intracranial occlusive disease. TEE showed right atrial SEC, a type $1 \mathrm{~B}$ atrial septal aneurysm, and no evidence of a right to left shunt by bubble contrast or color flow Doppler. The patient was treated with aspirin and has not had any recurrent neurologic events in four months of follow-up.

\section{Discussion}

Frequency and etiology of right atrial SEC

Right atrial SEC is an uncommon finding at TEE. In our study, only $1.2 \%$ of consecutive patients undergoing TEE had right atrial SEC whereas $7.7 \%$ of these patients had left atrial SEC. Previous reports have focused on left atrial SEC, with only brief mention of the clinical and echocardiographic features associated with right atrial SEC.

Castello et al. [1] reported five patients with right atrial SEC - one had mitral and tricuspid valve disease, one had transposition of the great arteries and tricuspid atresia corrected by the Fontan procedure, and three had coronary artery disease with secondary right ventricular dysfunction (one of these three patients had a right ventricular infarction). All five patients were in sinus rythm, three had significant tricuspid regurgitation, and none had right atrial thrombus. Right atrial size was significantly larger in the five patients with right atrial SEC than in patients without right atrial $\operatorname{SEC}(52 \pm 10$ vs $44 \pm 5 \mathrm{p}<0.01)$.

Black et al. [2] briefly described eight patients with right atrial contrast - six had atrial fibrillation, one had severe left ventricular dysfunction, and one had constrictive pericarditis. Chia et al. [9] described one patient with right sided cardiomyopathy, right atrial SEC, and right atrial thrombus.

The findings in our study suggest that right atrial enlargement, markedly elevated right ventricular pressure, atrial fibrillation, and tricuspid regurgitation are associated with right atrial SEC. Since it is relatively common to identify one or more of these factors at TEE in patients who do not have right atrial SEC, other factors may be involved in the genesis of SEC. A recent study showed that patients with SEC have elevated fibrinogen levels compared to patients without SEC [10] which suggests that both abnormal blood rheology and hemodynamic factors may be necessary conditions for SEC to develop.

Seven of our eight patients with right atrial SEC had tricuspid regurgitation that was attributed to dilation of the tricuspid annulus secondary to right ventricular and right atrial dilation. It is of interest that severe tricuspid regurgitation does not prevent right atrial SEC whereas severe mitral regurgitation tends to prevent left atrial SEC $[2,3]$. The explanation for these findings may relate to the higher pressure differential across the mitral valve compared to the tricuspid valve when these valves are incompetent [6] i.e. the high force of a regurgitant jet from left ventricle to left atrium is more likely to prevent stasis of blood than the lower force associated with regurgitation from right ventricle to right atrium.

\section{Thromboembolism and right atrial SEC}

The well established association of left atrial SEC and increased risk of stroke [1-3, 10,11] suggests that right atrial SEC may also be associated with thromboembolism. Although two of the patients in our study had thromboembolic events, the association of these events with right atrial SEC is uncertain. In patient 2 , we speculate that the massively enlarged right atrium with sluggish blood flow was the source of the patients pulmonary emboli since no other source was established. However, it is also possible that the right heart findings at echocardiography were secondary to pulmonary emboli (source uncertain) and severe pulmonary hypertension.

In patient 4 , the etiology of the left hemispheral TIAs was not large artery cerebrovascular occlusive disease since cerebral angiography was normal. The patient had right atrial SEC and a type IB atrial septal aneurysm that is usually associated with an interatrial shunt [7]. This raises the possibility that the etiology of patient 4's TIAs was paradoxical embolism, but we 
were unable to demonstrate an interatrial shunt. It is possible, however, that we may have missed a small shunt.

Some authors have suggested that paradoxical embolism through a right to left interatrial shunt may be a common mechanism of stroke in patients with cryptogenic stroke [12-14], however, other authors maintain that paradoxical embolism is rare and is usually associated with concomitant pulmonary embolism [15]. Patients with right atrial SEC, elevated right heart pressures, and a right to left shunt through an interatrial septal defect may be a subgroup at particularly high risk of paradoxical embolism. Three of eight patients in our study had these echocardiographic features, one of whom also had a right atrial thrombus. Nonetheless, none of these three patients suffered a systemic embolus prior to TEE. Prospective data on embolic risk in these three patients are not available because two were lost to follow up, and the other patient underwent repair of the septal defect. Further studies are needed to clarify the association, if any, of right atrial SEC and paradoxical embolism.

\section{References}

1. Castello R, Pearson AC, Labovitz AJ. Prevalence and clinical implications of atrial spontaneous contrast in patients undergoing transesophageal echocardiography. Am J Cardiol 1990; 65: 1149-53.

2. Black IW, Hopkins AP, Lee LCL, Walsh WF. Left atrial spontaneous echo contrast: A clinical and echocardiographic analysis. J Am Coll Cardiol 1991; 18: 398-404.

3. Chimowitz MI, DeGeorgia MA, Poole RM, Hepner A, Armstrong WF. Left atrial spontaneous echo contrast is highly associated with previous stroke in patients with atrial fibrillation or mitral stenosis. Stroke 1993; 24: 1015-9.
4. Sahn DJ, DeMaria A, Kisslo J, Weyman A. Recommendations regarding quantitation in M-mode echocardiogreaphy: Results of a survey of echocardiographic measurements. Circulation 1978; 58: 1072-83.

5. Castello R, Lenzen P, Aguirre F, Labovitz A. Variability in the quantitation of mitral regurgitation by Doppler color flow mapping: Comparison of transthoracic and transesophageal studies. J Am Coll Cardiol 1992; 20: 433-8.

6. Olson LJ, Tajik AJ. Echocardiographic evaluation of valvular heart disease. In: Marcus ML, Schelbert HR, Skorton DJ, Wolf GL (eds.) Cardiac imaging: a companion to Braunwald's heart disease. Philadelphia: WB Saunders Co., 1991, Chapter 21: 419-448.

7. Pearson AC, Nagelhout D, Castello R, Gomez CR, Labovitz AJ. Atrial septal aneurysm and stroke: A transesophageal echocardiographic study. J Am Coll Cardiol 1991; 18: 1223-9.

8. Hanley PC, Tajik AJ, Hynes JK, Edwards WD, Reeder GS, Hagler DJ, Seward JB. Diagnosis and classification of atrial septal aneurysm by two-dimensional echocardiography: Report of 80 consecutive cases. J Am Coll Cardiol 1985; 6: 1370-82.

9. Chia BL, Choo MH, Yan PC, Ee BK, Lee CN, Shears JH. Intra-atrial smoke like echoes and thrombi formation. Chest 1989; 95: 912-4.

10. Briley DP, Giraud G, Spear G, Coull BM, Edwards J, Clark WM. Spontaneous echo contrast, ischemic stroke, and risk of recurrent stroke. Ann Neurol 1992; 237 (Abstract).

11. Daniel WG, Nellessen U, Schroder E, Nonnast-Daniel B, Bednarski P, Nikutta P, Lichtlen PR. Left atrial spontaneous echo contrast in mitral valve disease: An indicator for an increased thromboembolic risk. J Am Coll Cardiol 1988; 11: 1204-11.

12. Lechat $P$, Mas JL, Lascault G, Loron $P$, Theard M, Klimczac M, Drobinski G, Thomas D, Grosgogeat Y. Prevalence of patent foramen ovale in patients with stroke. N Engl J Med 1988; 318 : 1148-52.

13. Harvey JR, Teague SM, Anderson JL, Voyles WF, Thadani U. Clinically silent atrial septal defects with evidence for cerebral embolization. Ann Intern Med 1986; 105: 695-7.

14. Di Tullio M, Sacco RL, Gopal A, Mohr JP, Homma S. Patent foramen ovale as a risk factor for cryptogenic stroke. Ann Intern Med 1992; 117: 461-5.

15. Falk RH. PFO or UFO? The role of a patent foramen ovale in cryptogenic stroke. Ann Intern Med 1991; 121: 1264-6. 